\title{
Bandwidth Enhancement and Beam Squint Reduction of Leaky Modes in a Uniaxially Anisotropic Meta-substrate
}

\author{
A. Shahvarpour*1, A. Alvarez Melcon ${ }^{2}$, and C. Caloz ${ }^{1}$ \\ ${ }^{1}$ Poly-Grames Research Center, École Polytechnique de Montréal, \\ Montréal, QC, H3T 1J4, Canada \\ 2 Technical University of Cartagena, 30202 Cartagena, Spain \\ E-mail: attieh.shahvarpour@polymtl.ca
}

\section{Introduction}

Meta-substrates $[1,2]$ are artificial dielectric substrates with unusual properties. In [2] an anisotropic meta-substrate was proposed with the same fully-space scanning capability as leaky-wave antennas based on composite right/left-handed (CRLH) patterned transmission line (TL) structures. This meta-substrate is a mushroom type structure [3] with uniaxially anisotropic properties, where the wires perpendicular to the air-substrate interface exhibit a Drude permittivity and the loops between the adjacent mushrooms exhibit a Lorentz permeability. This paper presents a rigorous spectral TL approach analysis [4] of this meta-substrate, which is constituted of the anisotropic medium of Fig.1a, with the constitutive parameter tensors,

$$
\overline{\bar{\varepsilon}}=\left(\begin{array}{ccc}
\varepsilon & 0 & 0 \\
0 & \varepsilon & 0 \\
0 & 0 & \varepsilon_{z}
\end{array}\right), \quad \overline{\bar{\mu}}=\left(\begin{array}{ccc}
\mu_{\rho} & 0 & 0 \\
0 & \mu_{\rho} & 0 \\
0 & 0 & \mu
\end{array}\right)
$$

and which is represented in Fig.1b. Moreover, it derives, using the transverse resonance techniques [5], the dispersion relations of the TM modes, which are the modes with the most significant effects of dispersion and anisotropy, and discusses the effects of anisotropy on its leaky modes.

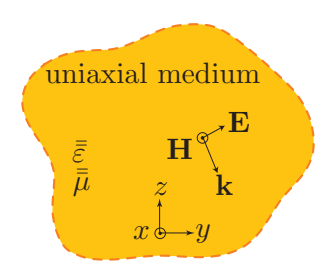

(a)

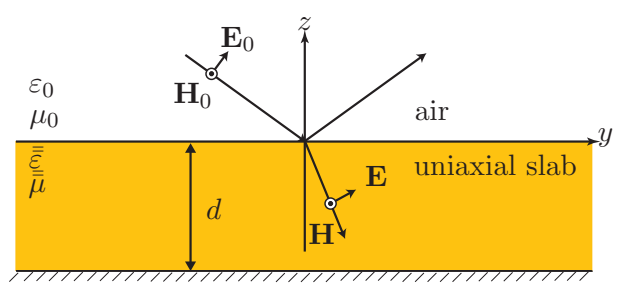

(b)

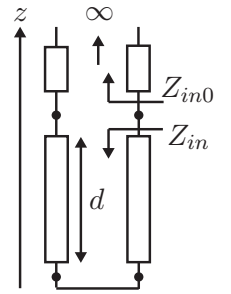

(c)

Figure 1: Uniaxially anisotropic structure. (a) Unbounded medium. (b) Grounded slab. (c) Transmission line (TL) model.

\section{TM Spectral TL Model for the Unbounded Anisotropic Medium}

Assuming the time dependence $\exp (+j \omega t)$, the $\mathrm{TM}_{z}$ spectral domain TL equations are relations between the electric field components in the form of $d \tilde{V} / d z=-j Z k_{z} \tilde{I}$ and $d \tilde{I} / d z=-j Y k_{z} \tilde{V}$, where $\tilde{V}, \tilde{I}, Z, Y$ and $k_{z}$ are the spectral voltage, current, impedance, admittance and the longitudinal wave number along the $z$ axis, 
respectively. The current solution of the TL equations is $\tilde{I}=1 / Z_{c}\left[\tilde{V}^{+} \exp (-j \beta z)-\right.$ $\left.\tilde{V}^{-} \exp (j \beta z)\right]$, where $\tilde{V}^{ \pm}$are constants, $Z_{c}=\sqrt{Z / Y}$ is the characteristic impedance and $\beta=\sqrt{Z Y} k_{z}$ is the propagation constant of the TL along the $z$ axis. The $\mathrm{TM}_{z}$ TL equations of the uniaxially grounded slab (Fig. 1b) are obtained by inserting Eq. (1) in the source-less spectral domain Maxwell's equations $\left(\tilde{H}_{z}=0\right)$, with $\partial / \partial x \rightarrow j k_{x}$ and $\partial / \partial y \rightarrow j k_{y}$, which leads to

$$
\begin{gathered}
-\frac{d \tilde{H}_{y}}{d z}=j \omega \varepsilon \tilde{E}_{x}, \\
\frac{d \tilde{H}_{x}}{d z}=j \omega \varepsilon \tilde{E}_{y}, \\
j k_{x} \tilde{H}_{y}-j k_{y} \tilde{H}_{x}=j \omega \varepsilon_{z} \tilde{E}_{z}, \\
j k_{y} \tilde{E}_{z}-\frac{d \tilde{E}_{y}}{d z}=-j \omega \mu_{\rho} \tilde{H}_{x}, \\
\frac{d \tilde{E}_{x}}{d z}-j k_{x} \tilde{E}_{z}=-j \omega \mu_{\rho} \tilde{H}_{y}, \\
j k_{x} \tilde{E}_{y}-j k_{y} \tilde{E}_{x}=0 .
\end{gathered}
$$

To find the first TL equation, derive Eq. (2c) with respect to $z$, then substitute Eqs. (2a) and (2b) into the resulting equation. Further eliminating $\tilde{E}_{y}$ by using Eq.(2f) leads to

$$
\frac{\partial}{\partial z}\left[-\frac{\omega \varepsilon_{z}}{k_{x}} \tilde{E}_{z}\right]=-j\left(\frac{\omega \varepsilon}{k_{z}}\right) k_{z}\left[-\frac{k_{\rho}{ }^{2}}{k_{x}{ }^{2}} \tilde{E}_{x}\right],
$$

where $k_{\rho}{ }^{2}=k_{x}{ }^{2}+k_{y}{ }^{2}$ is the square of the transverse wave number. For obtaining the second TL equation, substitute $\tilde{H}_{y}$ computed from Eq. (2c) into Eq. (2e) and substitute $\tilde{H}_{x}$ from Eq. (2d) into the resulting equation. Further eliminate $\tilde{E}_{y}$ from Eq. (2f), that leads to

$$
\frac{\partial}{\partial z}\left[-\frac{k_{\rho}^{2}}{k_{x}^{2}} \tilde{E}_{x}\right]=-j\left(\frac{k_{z}}{\omega \varepsilon_{z}}\right) k_{z}\left[\frac{-\omega \varepsilon_{z}}{k_{x}} \tilde{E}_{z}\right],
$$

where $k^{2}=k_{\rho}{ }^{2}+k_{z}{ }^{2}=\omega^{2} \mu_{\rho} \varepsilon_{z}$ is the square of the wave number. From Eqs. (3) and (4) the elements of the anisotropic TL model are found as $\tilde{V}=-\left(k_{\rho}{ }^{2} / k_{x}{ }^{2}\right) \tilde{E}_{x}, \tilde{I}=$ $-\left(\omega \varepsilon_{z} / k_{x}\right) \tilde{E}_{z}, Z=k_{z} / \omega \varepsilon_{z}, Y=\omega \varepsilon / k_{z}, Z_{c}=\left(k_{z} / \omega \varepsilon\right) \sqrt{\varepsilon / \varepsilon_{z}}$ and $\beta=\left(\sqrt{\varepsilon / \varepsilon_{z}}\right) k_{z}$.

\section{TM Dispersion Relation of the Uniaxially Anisotropic Slab}

The dispersion relation of the uniaxially grounded slab (Fig. 1b) with the TL model shown in Figs. 1c can be obtained by satisfying the transverse resonant condition $Z_{i n 0}+Z_{i n}=0$ [5], where $Z_{i n 0}=Z_{c 0}$ and $Z_{i n}=j Z_{c} \tan (\beta d)$ are the impedance of the semi-infinite free-space and the input impedance of the slab from its interface with the air, respectively, and $Z_{c 0}=k_{z 0} / \omega \varepsilon_{0}$ is the characteristic impedance of the free space with $k_{z 0}= \pm \sqrt{\omega^{2} \mu_{0} \varepsilon_{0}-k_{\rho}^{2}}$ as the longitudinal wave number in the air, that leads to

$$
j \frac{\sqrt{\omega^{2} \mu_{\rho} \varepsilon_{z}-k_{\rho}^{2}}}{\omega \varepsilon} \sqrt{\frac{\varepsilon}{\varepsilon_{z}}} \tan \left(\sqrt{\frac{\varepsilon}{\varepsilon_{z}}} \sqrt{\omega^{2} \mu_{\rho} \varepsilon_{z}-k_{\rho}^{2}} d\right) \pm \frac{\sqrt{\omega^{2} \mu_{0} \varepsilon_{0}-k_{\rho}^{2}}}{\omega \varepsilon_{0}}=0 .
$$


Table 1: Characteristics of the $\mathrm{TM}_{z}$ modes of the isotropic grounded slab (Fig. 2).

\begin{tabular}{c|ccccc} 
Mode & $\operatorname{Re}\left(k_{\rho} / k_{0}\right)$ & $\operatorname{Im}\left(k_{\rho} / k_{0}\right)$ & $\operatorname{Re}\left(k_{z 0} / k_{0}\right)$ & $\operatorname{Im}\left(k_{z 0} / k_{0}\right)$ & Nature \\
\hline a & $1<$ and $<\sqrt{\varepsilon}$ & $=0$ & $=0$ & $<0$ & surface mode \\
b, c & $1<$ and $<\sqrt{\varepsilon}$ & $=0$ & $=0$ & $>0$ & non-physical \\
d, f & $>1$ & $<0$ & $>0$ & $>0$ & non-physical \\
e & $<1$ & $<0$ & $>0$ & $>0$ & leaky mode
\end{tabular}

\section{Dispersion Analysis}

Fig. 2 compares the dispersion behavior of the anisotropic slab with various amounts of anisotropy $\varepsilon_{z} / \varepsilon\left(\mu=\mu_{\rho}=\mu_{0}\right)$ with that of an isotropic slab with $\varepsilon=2 \varepsilon_{0}$ and $\mu=\mu_{0}$. The modes of the isotropic slab [6] are summarized in Tab. 1. It is seen that decreasing $\varepsilon_{z} / \varepsilon$ decreases the bandwidth of the surface modes and transform them into leaky modes, especially for $\mu_{\rho} \varepsilon_{z}<1$. Moreover, the slopes of the leaky modes decrease and become almost flat for a sufficient amount of electrical thickness. This provides the following practical benefit: since the radiation angle of the main beam of a leaky-wave antenna is $\theta(\omega)=\sin ^{-1}\left(k_{\rho} / k_{0}\right)$, a flat dispersion curve (i.e. a fixed $\left.k_{\rho} / k_{0}\right)$ results in a fixed pointing angle, immune of beam squint. In addition, the bandwidth of the leaky-mode with fixed pointing angle is enhanced.

However, the anisotropic meta-substrate of interest is inherently dispersive [2]. The wires along the $z$ axis exhibit a Drude permittivity along this axis, $\varepsilon_{z} / \varepsilon_{0}=$ $\varepsilon_{r \infty}-\omega_{e p}{ }^{2} / \omega^{2}[7]$, while the loops between the adjacent mushrooms exhibit a Lorentz permeability along the $\rho$ axis, $\mu_{\rho} / \mu_{0}=1-F \omega^{2} /\left(\omega^{2}-\omega_{m 0}{ }^{2}\right)$ [8]. The permittivity and permeability along the other axes are the same as for the host medium. Fig. 3 shows the curves of $\varepsilon_{z} / \varepsilon_{0}$ and $\mu_{\rho} / \mu_{0}$ and the dispersion characteristics of the dispersive anisotropic slab. In this study, only the right-handed frequency range is analyzed. The figure shows that for sufficient amount of electric thickness, the dispersive anisotropic grounded slab shows the same characteristics as the non-dispersive slab.

\section{Conclusion}

The TM dispersion characteristic of a uniaxially anisotropic meta-substrate has been analyzed by the spectral domain transmission line (TL) approach. It has been shown that the beam squint effect in its leaky-modes may be significantly reduced and that the bandwidth of these leaky-modes may become very large. This effect may lead to unprecedented applications in point-to-point communication and sensing applications.

\section{References}

[1] H. Mosallaei and K. Sarabandi, "Design and modeling of patch antenna printed on magneto-dielectric embedded-circuit metasubstrate," IEEE Trans. Antennas Propagat., vol. 55, no. 1, pp. 45-52, Jan. 2007.

[2] H. V. Nguyen and C. Caloz, "Anisotropic backward-wave meta-susbtrate and its application to a microstrip leaky-wave antenna," in Proc. CNC/USNC URSI National Radio Science Meeting, Ottawa, ON, Canada, July 2007. 


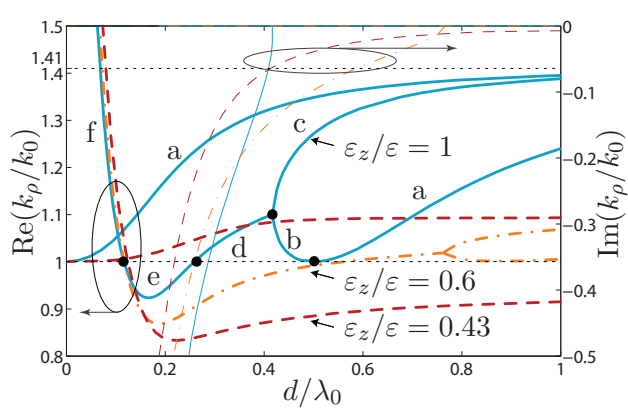

(a)

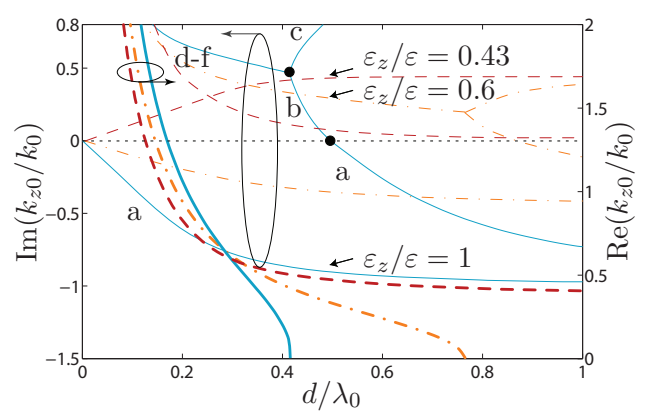

(b)

Figure 2: Dispersion of the various anisotropic slabs with $\varepsilon=2 \varepsilon_{0}$ and $\mu=\mu_{\rho}=\mu_{0}$, for the spatial dependencies $\exp \left(-j \mathbf{k}_{0} \cdot \mathbf{r}\right)=$ $\exp \left(-j \operatorname{Re}\left(k_{\rho}\right) \rho\right) \exp \left(\operatorname{Im}\left(k_{\rho}\right) \rho\right) \exp \left(-j \operatorname{Re}\left(k_{z 0}\right) z\right) \exp \left(\operatorname{Im}\left(k_{z 0}\right) z\right) . \quad$ (a) $\operatorname{Re}\left(k_{\rho} / k_{0}\right) \quad$ and $\operatorname{Im}\left(k_{\rho} / k_{0}\right)$. (b) $\operatorname{Re}\left(k_{z 0} / k_{0}\right)$ and $\operatorname{Im}\left(k_{z 0} / k_{0}\right)$.

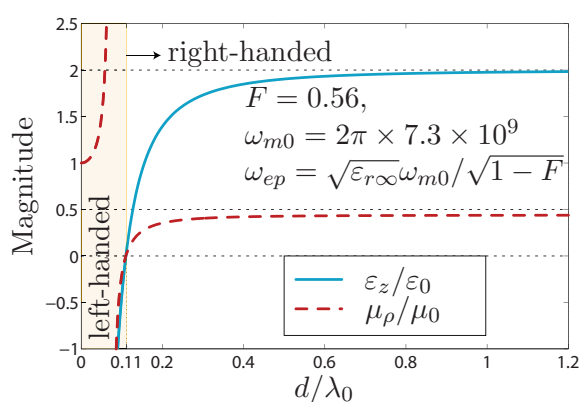

(a)

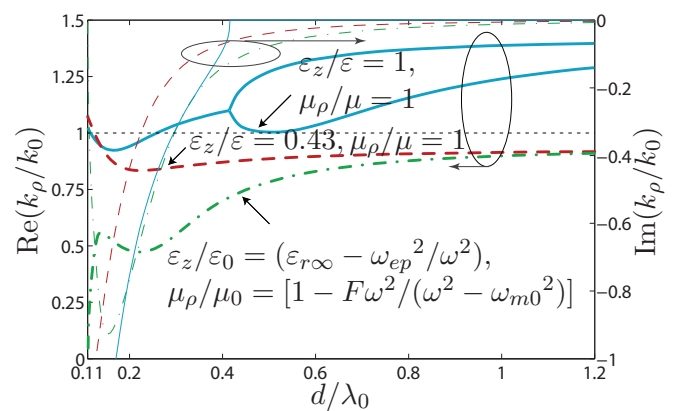

(b)

Figure 3: Dispersion curves of the dispersive and non-dispersive anisotropic grounded slab's leaky-mode. (a) $\varepsilon_{z} / \varepsilon_{0}$ and $\mu_{\rho} / \mu_{0}$ dispersion curves . (b) $\operatorname{Re}\left(k_{\rho} / k_{0}\right)$ and $\operatorname{Im}\left(k_{\rho} / k_{0}\right)$.

[3] D. Sievenpiper, L. Zhang, R. F. J. Broas, N. G. Alexopolous, and E. Yablonovitch, "High-impedance electromagnetic surfaces with forbidden frequency band," IEEE Trans. Microwave Theory Tech., vol. 47, no. 11, pp. 20592074, Nov. 1999.

[4] L. B. Felsen and N. Marcuvitz, Radiation and scattering of Waves, PrenticeHall/IEEE Press, NY, 1996.

[5] N. Marcuvitz (Ed.), Waveguide Handbook, chapter 8, McGraw-Hill, NY, 1951.

[6] D. Jackson and A. Oliner, chap. 7 in C. A. Balanis (editor), Modern Antenna Handbook, John Wiley, Inc., 2008.

[7] S. A. Tretyakov, Analyitical Modeling in Applied Electromagnetics, Artech House, Norwood, MA, 2003.

[8] J. B. Pendry, A. J. Holden, D. J. Robbins, and W. J. Stewart, "Magnetism from conductors and enhanced non-linear phenomena," IEEE Trans. Microwave Theory Tech., vol. 47, no. 11, pp. 2075-2084, Nov. 1999. 